\title{
Management of rheumatic atrial fibrillation
}

\begin{tabular}{l|l}
\hline Ashley Chin & ABSTRACT \\
\hline $\begin{array}{l}\text { Cardiac Clinic, Groote Schuur Hospital, University of Cape Town, } \\
\text { Observatory, South Africa }\end{array}$ & $\begin{array}{l}\text { Thromboembolism, secondary to rheumatic atrial fibril- } \\
\text { lation, remains an important cause of stroke and systemic } \\
\text { embolism in low and middle income countries. This } \\
\text { review will highlight the pathogenesis of rheumatic atrial }\end{array}$ \\
\hline Address for correspondence: & $\begin{array}{l}\text { fibrillation and thromboembolism. The risk factors and } \\
\text { prevention of thromboembolism, including management }\end{array}$ \\
Ashley Chin & $\begin{array}{l}\text { strategies of rheumatic atrial fibrillation, will also be } \\
\text { Cardiac Clinic }\end{array}$ \\
Groote Schuur Hospital & Unived. SAHeart $2015 ; 12: 152-155$
\end{tabular}

University of Cape Town

Main Road

Observatory

Cape Town

7925

South Africa

Email:

ashley.chin@uct.ac.za

\section{INTRODUCTION}

Rheumatic heart disease (RHD) is an important cause of morbidity and mortality worldwide. (I) In low and middle income countries, the incidence of acute rheumatic fever (ARF) remains relatively high (>I per 10000 people per year). (2) The prevalence of chronic RHD, the legacy of ARF, continues to rise in all regions of the world, except Europe and parts of the Americas. ${ }^{(3)}$

Atrial fibrillation (AF) is a major complication of chronic RHD. AF develops in approximately 18 - 39\% of RHD patients and increases the risk of thromboembolism. (4,5) In low and middle income regions, like Africa and India, RHD still accounts for 22 - 32\% of all AF patients presenting to an emergency room and $3-8 \%$ of all strokes in China. ${ }^{(6,7)}$

This review will highlight the pathogenesis and epidemiology of $\mathrm{RHD}, \mathrm{AF}$ and thromboembolism and discuss the evidence for antithrombotic therapy and rhythm control strategies.

\section{PATHOGENESIS OF RHD, AF AND THROMBOEMBOLISM}

Acute rheumatic fever (ARF) is caused by a group A streptococcal infection of the tonsillo-pharynx. ${ }^{(8)}$ Molecular mimicry between streptococcal $M$ proteins and host tissue results in auto-antibody formation against cardiac endothelium, lamina, and the laminar basement membrane which results in a pancarditis and fibrosis of the heart valves. Mitral valve disease (particularly mitral stenosis (MS)) increases left atrial (LA) pres- sure and volume which increases LA size and promotes blood stasis. Loss of coordinated LA contraction further leads to blood stasis in the LA and left atrial appendage (LAA) which predisposes to thrombus formation (Figure I). LA inflammation, atrial fibrosis and myocyte atrophy result in shortening of the atrial action potential and refractory periods which predispose to re-entrant circuits and AF (Figure 2). ${ }^{(9)}$

Increasing LA size and multivalvar involvement have been shown to be important clinical predictors of AF and thrombus formation. ${ }^{(4,10,11)}$ The mean reported $L A$ sizes ranged from $4.3-5.7 \mathrm{~cm}$ in these studies. In Wood's series, AF occurred in $29 \%$ of patients with isolated MS compared to $70 \%$ of patients with mixed mitral valve disease and tricuspid regurgitation. Valve severity, determined by mitral valve area at

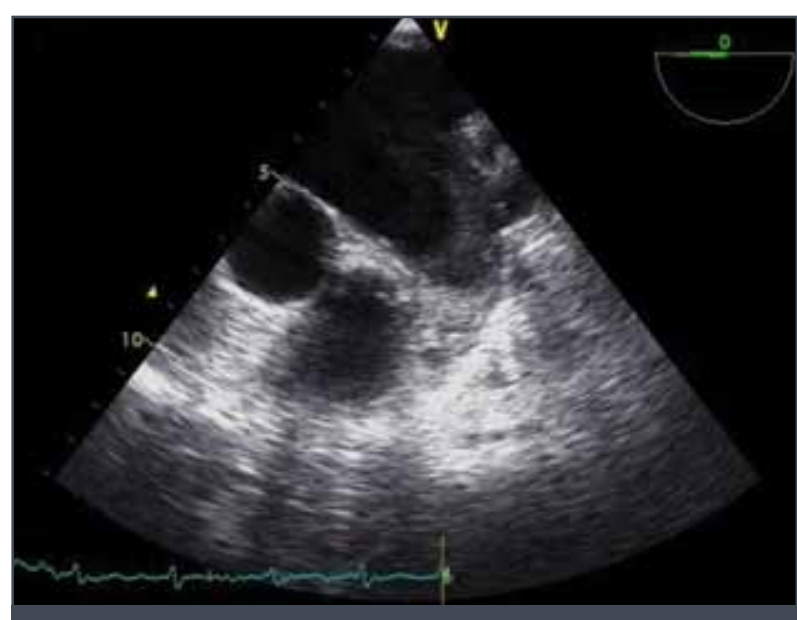

FIGURE I: A transesophageal echocardiogram of a patient with mitral stenosis and atrial fibrillation.

This high-oesophageal view shows a large thrombus in the left atrial appendage with spontaneous echo contrast in the enlarged left atrium. 


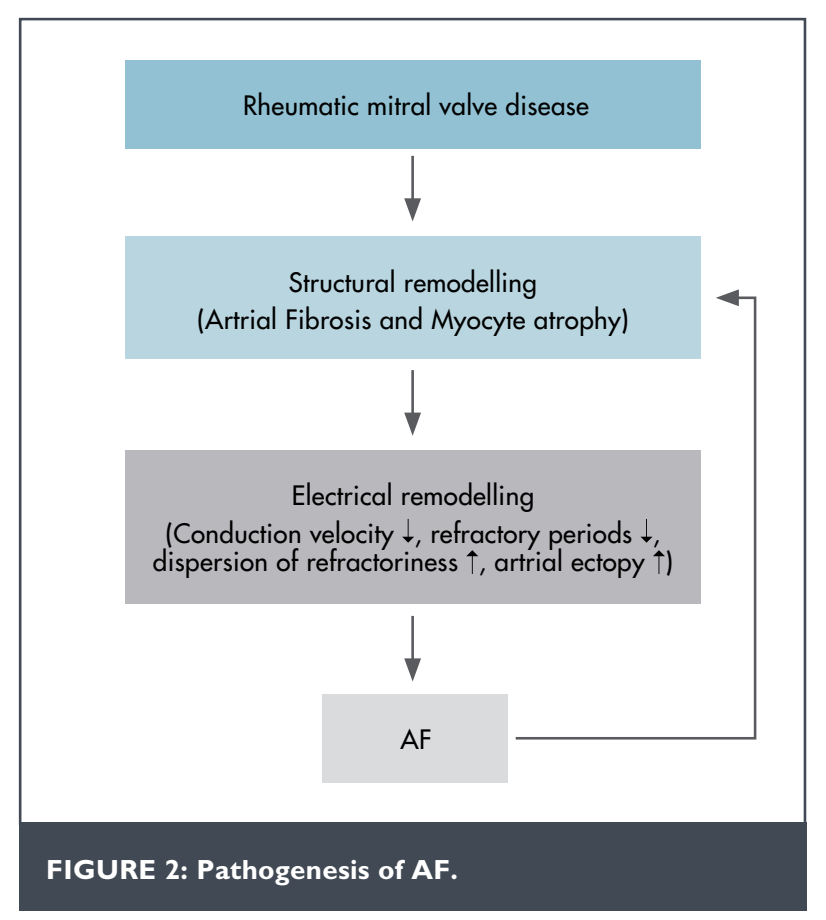

cardiac catheterisation, or mean diastolic gradient by echocardiogram were not predictors of the development of $A F$ in any of these studies.

\section{RISK OF THROMBOEMBOLISM IN AF}

The risk of rheumatic AF and thromboembolism has always been thought to be high, based on old natural history studies of MS. In 2 old retrospective studies of MS and AF, thromboemboli occurred in 22 - 32\% of all patients with MS and AF compared to 6 - $8 \%$ of patients with MS and sinus rhythm. ${ }^{(1,12)}$ In Wood's series, thromboembolic episodes were cerebral in $75 \%$, peripheral in 33\% and visceral in 6\%; $22 \%$ of patients had both cerebral and peripheral emboli. It was also noted that MS carried a higher risk (HR I.5) of thromboembolism compared to mitral regurgitation (MR). This was also observed by Coulshed who reported that $32 \%$ of MS cases with AF develop thromboembolism compared to $22 \%$ of MR cases with AF between I and 13 years of follow-up.

There have been 2 old prospective studies of RHD which have reported incidence rates of thromboembolism in patients with AF. ${ }^{(13,14)}$ Szekely reported a 7-fold increase in thromboembolism (5\% per year) with AF compared to patients without AF $(0.7 \%$ per year). The majority of thromboembolic episodes were first events and antithrombotic therapy was used in the minority (33\%) of patients who had had a prior thromboembolism. Analysis from a Framingham cohort reported a stroke incidence of $4.5 \%$ per year with AF and RHD which was a 17-fold increase compared to an age, sex and hypertension-matched population without AF and RHD. This study likely underestimated the true number and incidence of strokes, because AF was only detected through biennial ECGs and strokes discovered prior to the diagnosis of AF were excluded.
Traditional $\mathrm{CHADS}_{2}$ score risk factors of stroke (heart failure, hypertension, age $>75$ years, diabetes and prior stroke or systemic thromboembolism) have never been tested in the valvular AF population. ${ }^{(15)}$ A contemporary Chinese study of I 30 patients with RHD and stroke ( $82 \%$ had AF) reported a high recurrence rate of stroke of $13.6 \%$ per year. . $^{(7)}$ Based on these old studies, RHD and AF carries a risk of thromboembolism equivalent to a non-valvular AF population of $\mathrm{CHADS}_{2}$ score of 2 or 3; while this risk increases considerably with a prior history of TIA/CVA or systemic embolism.

\section{RISK OF THROMBOEMBOLISM IN SINUS RHYTHM}

It is well known that MS patients in sinus rhythm are also at risk of thromboembolism. A recent systematic review revealed 6 studies which reported thromboembolic rates in sinus rhythm of $0.5-5.3 \%$ per year. ${ }^{(16)}$ The rates from 4 retrospective studies were lower than the 2 prospective studies because of the inherent limitation of underreporting in retrospective studies. A study by Chiang showed that thromboembolism occurred in $9 \%$ of patients with sinus rhythm compared to 13\% of patients in AF. ${ }^{(17)}$ Risk factors for thromboembolism in this study included increasing age, presence of LA thrombus, decreasing mitral valve area and significant aortic regurgitation. Other echocardiographic predictors of thromboemboli include the presence of spontaneous echo contrast and a reduced LAA emptying flow velocity. ${ }^{(18,19)}$

In a recent prospective study of $M S$ and sinus rhythm, transient subclinical AF detected by a single Holter monitor was detected in $27 \%$ of cases and was an independent predictor of systemic embolism with a crude embolic rate of $5.3 \%$ per year. ${ }^{(16)}$ It is likely that subclinical AF is higher in clinical practice as monitoring for AF was limited to 24 hours in this study. Pending analyses of the REMEDY and RE-LY AF studies will give us further insights into the risk of thromboembolism in the RHD AF and sinus rhythm population.

\section{PREVENTION OF THROMBOEMBOLISM}

Secondary prevention

Antithrombotic (vitamin $\mathrm{K}$ antagonist (VKA)) therapy has been widely used since the 1970s to decrease the risk of recurrent thromboembolism in RHD patients (secondary prevention). Although observational studies support the use of antithrombotic therapy, there have been no randomised trials conducted in this population. ${ }^{(20,21)}$ Nevertheless, VKA therapy is clearly indicated in this population because of the high risk of recurrent thromboembolism.

\section{Primary prevention}

Antithrombotic therapy is recommended to prevent thromboembolism in patients with AF without a prior thromboembolism (primary prevention). As RHD was an exclusion criterion for all of the contemporary AF trials, recommendations are largely based on extrapolation of randomised placebo-controlled trials of nonvalvular AF. Although these trials showed a 
marked reduction in thromboembolism in patients taking VKAs, a similar reduction in risk of thromboembolism in patients with RHD cannot be assumed. Despite the inherent bias of nonrandomised studies and the weak methodologies reflecting practices at the time, antithrombotic therapy appeared to reduce the thromboembolic risk in two observational studies of RHD and AF. Fleming reported a low rate of thromboemboli (5 of 217 patients $(0.8 \%)$ ) in patients taking warfarin. ${ }^{(22)}$ Another study of 254 patients with AF (including 120 patients with mitral valve disease) reported a thromboembolic risk of $5.46 \%$ per year without warfarin versus $0.7 \%$ per year for those taking warfarin. ${ }^{(23)}$

There are unique challenges facing the clinician when prescribing antithrombotic therapy in the developing world. Non-compliance to antithrombotic therapy and lack of INR monitoring in impoverished settings can increase risks of major bleeding and reduce the efficacy of warfarin. ${ }^{(24)}$ Furthermore, for patients taking warfarin, percentage of INR values in the therapeutic range is also unsatisfactory $(<40 \%){ }^{(6)}$ Potential warfarin teratogenicity is also a major concern for women of childbearing age.

There have been no antithrombotic studies in patients with RHD and sinus rhythm. Although risk factors like increasing age, LA thrombus, spontaneous echo contrast and an enlarged LA have been identified, it remains unclear whether therapy is effective in these subgroups. ${ }^{(17)}$ As the majority of RHD patients are in sinus rhythm and are potentially at risk, future trials in this population group are clearly warranted.

\section{WHAT DO THE GUIDELINES RECOMMEND?}

The current recommendations for antithrombotic therapy and RHD are summarised in Table I. These guidelines are based on limited scientific evidence.

The AHA/ACC 2014 AF guidelines have classified MS as a "high risk" condition for thromboembolism, but no specific recommendations are made in this guideline. ${ }^{(25)}$ The ESC 2010 AF guidelines also classify MS as a "high risk" condition and recommend warfarin therapy (class IA). ${ }^{(26)}$ The AHA/ACC 2014 valvular heart disease guidelines recommend warfarin in patients for $M S$ and $A F$, prior embolic stroke in sinus rhythm and/or left atrial thrombus (Class IB).(27) These guidelines also state that warfarin for asymptomatic patients with severe MS and LA dimension $>55 \mathrm{~mm}$, or enlarged LA with spontaneous echo contrast on echocardiogram, is controversial.

These guidelines do not specify recommendations for patients with RHD and MR or aortic regurgitation, but these patients should probably be considered an MS equivalent. There have been no trials of any of the novel oral anti-coagulants (NOACs) in haemodynamically-significant valvular AF and none can be recommended at this time. Studies of the NOACs (e.g. dabigatran, rivaroxaban and apixaban) have all excluded patients with prosthetic heart valves and mitral stenosis. In non-valvular AF these agents have shown similar or superior reduction in thromboembolic rates compared to warfarin and a better safety profile. These agents are particularly attractive in a RHD population as INR monitoring is not required. Despite the high costs of these drugs and the lack of efficacy in patients with prosthetic heart valves, the NOACS have the potential to improve patient outcomes in the RHD population and future trials are needed.

\section{RATE VERSUS RHYTHM CONTROL IN RHEUMATIC AF}

The landmark randomised trials of rate versus rhythm control did not include patients with rheumatic AF. ${ }^{(28,29)}$ In the AFFIRM trial, $<5 \%$ of patients had valvular heart disease. A rate control strategy (using beta-blockers, calcium blockers and/or digoxin) is usually adopted in clinical practice because of the low likelihood of maintaining sinus rhythm in the long-term. For patients with very symptomatic paroxysmal AF, a rhythm control strategy using cardioversion and amiodarone can be used. A small, underpowered randomised trial of rheumatic $A F$, using amiodarone as a rhythm control strategy, maintained sinus rhythm in $69 \%$ of patients at I year. ${ }^{(30)}$ The poor long-term tolerability of amiodarone, and the inability to use class I antiarrhythmic drugs (like flecainide) in patients with underlying left ventricular dysfunction, often preclude the use of these drugs to maintain sinus rhythm.

A long-term observational study from a single center has reported that the Cox-maze III procedure at the time of mitral valve surgery is effective in reducing AF over long term followup. (31) With the surgical complexity of the Cox-Maze III procedure, surgical catheter ablation using saline irrigated catheter ablation (SICTRA) has become more attractive. A single randomised control trial showed that SICTRA was effective

TABLE I: Summary of major guidelines for antithrombotic therapy and RHD.

\begin{tabular}{|c|c|c|}
\hline \multicolumn{3}{|l|}{ Atrial fibrillation } \\
\hline $\begin{array}{l}\text { AHA/ACC/HRS } 2014 \\
\text { AF guidelines } \\
\text { (January, et al.) }\end{array}$ & $\begin{array}{l}\text { Mitral stenosis } \\
\text { classified as a high risk } \\
\text { condition }\end{array}$ & $\begin{array}{l}\text { No specific } \\
\text { recommendation }\end{array}$ \\
\hline $\begin{array}{l}\text { ESC 20I0 AF } \\
\text { guidelines } \\
\text { (Camm, et al.) }\end{array}$ & Mitral stenosis & Warfarin (Class I, A) \\
\hline $\begin{array}{l}\text { AHA/ACC } 2014 \\
\text { Valvular Heart Disease } \\
\text { guidelines } \\
\text { (Nishimura, et al.) }\end{array}$ & Mitral stenosis & Warfarin (Class I, B) \\
\hline \multicolumn{3}{|l|}{ Sinus rhythm } \\
\hline $\begin{array}{l}\text { AHAVACC } 2014 \\
\text { Valvular Heart Disease } \\
\text { guidelines } \\
\text { (Nishimura, et al.) }\end{array}$ & $\begin{array}{l}\text { Mitral stenosis } \\
\text { - Prior stroke } \\
\text { - LA thrombus } \\
\text { - LA > =55mm } \\
\text { (severe MS) } \\
\text { - Spontaneous echo } \\
\text { contrast }\end{array}$ & $\begin{array}{l}\text { Warfarin (Class I, B) } \\
\text { Warfarin (Class I, B) } \\
\text { Controversial } \\
\text { Controversial }\end{array}$ \\
\hline
\end{tabular}


in restoring sinus rhythm in patients undergoing mitral valve replacement without a reduction in survival. ${ }^{(32)} \mathrm{A}$ randomised trial of SICTRA at the time of mitral valve surgery was superior to conventional catheter ablation (pulmonary vein isolation) at 6 months post surgery in reducing recurrences of $A F^{(33)}$ Until multicentre trials of catheter ablation in rheumatic AF show clinical benefit, catheter ablation of rheumatic AF remains investigational.

\section{CONCLUSION}

Rheumatic AF and thromboembolism remain a major cause of morbidity and mortality in low and middle income countries. Although anticoagulation to prevent thromboembolism is indicated in all patients with rheumatic AF with a low bleeding risk, there are unique challenges in prescribing VKA in these regions. The safety and efficacy of the NOACs in rheumatic AF needs to be explored. Contemporary prospective studies on thromboembolic risk in RHD patients in AF and sinus rhythm are required.

\section{Conflict of interest: none declared.}

\section{REFERENCES}

।. Lozano R, et al. Global and regional mortality from 235 causes of death for 20 age groups in 1990 and 2010: A systematic analysis for the global burden of disease study 20 I0. Lancet 20 I 2;380(9859):2095- 128.

2. Tibazarwa KB, et al. Incidence of acute rheumatic fever in the world: $A$ systematic review of population-based studies. Heart 2008;94(I2): I534-40.

3. Seckeler MD, et al. The worldwide epidemiology of acute rheumatic fever and rheumatic heart disease. Clin Epidemiol 201 1;3:67-84.

4. Diker $\mathrm{E}$, et al. Prevalence and predictors of atrial fibrillation in rheumatic valvular heart disease. Am J Cardiol 1996;77(1):96-8.

5. Zuhlke $\mathrm{L}$, et al. Characteristics, complications, and gaps in evidence-based interventions in rheumatic heart disease: The Global Rheumatic Heart Disease Registry (the REMEDY study). Eur Heart J 20 | 4;36( | 8): | | I 5-22.

6. Oldgren J, et al. Variations in cause and management of atrial fibrillation in a prospective registry of 15400 emergency department patients in 46 countries: The RE-LY Atrial Fibrillation Registry. Circulation 2014;129(I5): 1568-76.

7. Wang $D$, et al. Features of acute ischaemic stroke with rheumatic heart disease in a hospitalised Chinese population. Stroke 2012;43(1 I):2853-7.

8. Cunningham MW. Streptococcus and rheumatic fever. Curr Opin Rheumatol 20 12;24(4):408-16.

9. Kourliouros $\mathrm{A}$, et al. Current concepts in the pathogenesis of atrial fibrillation. Am Heart J 2009;157(2):243-52.

10. Ozaydin M, et al. Factors associated with the development of atrial fibrillation in patients with rheumatic mitral stenosis. Int I Cardiovasc Imaging 2010;26(5):547-52.

11. Wood P. An appreciation of mitral stenosis. I. Clinical features. Br Med J 1954; I (4870): 105 I-63.

12. Coulshed $\mathrm{N}$, et al. Systemic embolism in mitral valve disease. Br Heart J 1970;32(I):26-34.

13. Szekely P, et al. Rheumatic fever and rheumatic heart disease; natural history and preventive aspects. public health 1964;78:78-84
14. Wolf PA, et al. Epidemiologic assessment of chronic atrial fibrillation and risk of stroke: The Framingham study. Neurology 1978;28(10):973-7.

15. Gage BF, et al. Validation of clinical classification schemes for predicting stroke: Results from the National Registry of Atrial Fibrillation. JAMA 2001;285(22):2864-70.

16. Karthikeyan G, et al. Transient, subclinical atrial fibrillation and risk of systemic embolism in patients with rheumatic mitral stenosis in sinus rhythm. Am J Cardiol, 20 |4; | |4(6):869-74.

17. Chiang $\mathrm{CW}$, et al. Predictors of systemic embolism in patients with mitral stenosis. A prospective study. Ann Intern Med 1998; 128(1 I):885-9.

18. Li YH, et al. Importance of left atrial appendage function as a risk factor for systemic thromboembolism in patients with rheumatic mitral valve disease. Am J Cardiol 1996;78(7):844-7.

19. Acarturk E, et al. Thromboembolism risk in patients with mitral stenosis. Jpn Heart J 1997;38(5):669-75.

20. Szekely P. Systemic embolism and anticoagulant prophylaxis in rheumatic heart disease. Br Med J 1964; I (5392): I 209- 1212.

21. Wood JC, et al. Prevention of systemic arterial embolism in chronic rheumatic heart disease by means of protracted anticoagulant therapy. Circulation 1954;10(4):517-523.

22. Fleming HA. Anticoagulants in rheumatic heart-disease. Lancet 1971; 2(7722):486

23. Roy $D$, et al. Usefulness of anticoagulant therapy in the prevention of embolic complications of atrial fibrillation. Am Heart J 1986; I I 2(5): 1039-43.

24. Buchanan-Lee B, et al. Fixed-dose versus adjusted-dose warfarin in patients with prosthetic heart valves in a peri-urban impoverished population. J Heart Valve Dis 2002; I I (4):583-92.

25. January $C T$, et al. 2014 AHA/ACC/HRS guideline for the management of patients with atrial fibrillation: Executive summary: A report of the American College of Cardiology/American Heart Association task force on practice guidelines and the Heart Rhythm Society. Circulation 20 I 4; I 30(23):207 I - 104.

26. Camm AJ, et al. Guidelines for the management of atrial fibrillation: The Task Force for the Management of Atrial Fibrillation of the European Society of Cardiology (ESC). Eur Heart J 201 1;31 (19):2369-429.

27. Nishimura RA, et al. 2014 AHA/ACC Guideline for the management of patients with valvular heart disease: A report of the American College of Cardiology/American Heart Association task force on practice guidelines. Circulation 2014;129(23):521-643.

28. Wyse DG, et al. A comparison of rate control and rhythm control in patients with atrial fibrillation. N Engl J Med 2002;347(23): I 825-33.

29. Van Gelder IC, et al. A comparison of rate control and rhythm control in patients with recurrent persistent atrial fibrillation. N Engl J Med 2002; 347(23): 1834-40.

30. Vora $A$, et al. Control of rate versus rhythm in rheumatic atrial fibrillation: A randomised study. Indian Heart J 2004;56(2): I 10-6.

31. Kim KC, et al. Long-term results of the Cox-Maze III procedure for persistent atrial fibrillation associated with rheumatic mitral valve disease: 10-year experience. Eur J Cardiothorac Surg 2007;3I (2):26I-6.

32. Abreu Filho CA, et al. Effectiveness of the maze procedure using cooled-tip radiofrequency ablation in patients with permanent atrial fibrillation and rheumatic mitral valve disease. Circulation 2005; I 2 (Suppl 9):120-5.

33. Liu $X$, et al. Efficacy of catheter ablation and surgical CryoMaze procedure in patients with long-lasting persistent atrial fibrillation and rheumatic heart disease: a randomised trial. Eur Heart J 2010;31 (21):2633-4I. 